\title{
STUDY ON THE DETERMINATION OF COMBINING ABILITIES OF INBRED LINES FOR HYBRID BREEDING USING LINE $\times$ TESTER ANALYSIS IN SUNFLOWER (Helianthus annuus L.)
}

\author{
Tan, A.S. ${ }^{*}$
}

Ministry of Agriculture and Rural Affairs, General Directorate of Agricultural Research, Aegean Agricultural Research Institute, Plant Genetic Resources Department, P.O. Box. 9, Menemen, 35661 Izmir, Turkey

Received: April 10, 2010

Accepted: August 10, 2010

\section{SUMMARY}

Combining ability studies in oilseed sunflower were undertaken with a set of $5 \times 4$ line $\times$ tester including parents for the characters seed yield, 1000seed weight, days to flowering, days to physiological maturity, plant height, head diameter, stem diameter, oil content, fatty acid content (oleic, linoleic, palmitic, and stearic acids), protein content, seed length, seed width, and hull percentage. General (GCA) and specific combining abilities (SCA) and heterosis of inbred lines and their hybrids were estimated in a line $\times$ tester analysis during the first and second crop production seasons in Menemen, Izmir, Turkey. The variances due to GCA and SCA were highly significant for most of the characters in both environments. The ratio (H/D) $1 / 2$ and $\sigma^{2}$ GCA $\sigma^{2}$ SCA depicted the preponderance of non-additive type gene action for all the characters except plant height, head diameter, seed length, palmitic acid content, and stearic acid content. However, both types of gene action were observed for seed yield, hull percentage, 1000-seed weight, oil content, and stem diameter at stem curve point. In this study, GCA effects were found to be highly significant for all traits, while SCA effects were non-significant for most of the traits. Based on GCA effects in the first and second crop production seasons, the inbreds $0043 \mathrm{cms}, 0046 \mathrm{cms}, 0195 \mathrm{cms}, 0583 \mathrm{cms}, 0704 \mathrm{cms}, 0708 \mathrm{Rf}, 0845$ $R f$, $0951 R f$, and $1097 R f$ exhibited desirable GCA effects and were found to be good general combiners for most of the traits. Thus, they can be exploited by further breeding for developing superior genotypes and hybrids in sunflower.

Key words: sunflower, Helianthus annuus L., hybrid, breeding, genetics, general combining ability (GCA), specific combining ability (SCA), heterosis

* Corresponding author: Phone: +902328461331/470; e-mail: a_s_tan@hotmail.com 


\section{INTRODUCTION}

As the leading product in the production of oilseeds with great importance in human nutrition, sunflower has a significant place in the Turkish economy. Our vegetable oil deficit, which is increasing with the rapid population growth, will only be closed via increased production, which is possible, in turn, through the utilization of the existing potential area, the preference of sunflower specifically as a secondary product, and the application of new technologies. In hybrid breeding, grain and oil yields are increased by using the heterosis effect. The high performance of hybrid combinations depends on the combining abilities of parents and success in combination breeding is secured via the selection of the right parents (Tan, 1993; Tan, 2000; Tan, 2004; Tan, 2005; Tan, 2009). Therefore, parents must be examined in terms especially of economically significant properties to select the right ones to form hybrids. Properties addressed for heritability in plant breeding are also significant in terms of selection feasibility. As a general definition, heritability is defined as the ratio of total genotypic variance (encompassing additive, dominant and epistatic variances) to phenotypic variance (Kemptorne, 1957; Falconer, 1975). In the narrow sense, heritability is the ratio of additive variance (which is of great importance in terms of the properties to be transferred to the next generation) to the total variance (Falconer, 1975). Grain yield in sunflower depends on a number of factors, most notably the environment.

Plant breeders aim to reach the maximum level of heterosis during hybridization. Hallauer and Miranda (1981) divided heterosis-related studies into two categories:

a) physiological impulse, allelic interaction or over-dominance, and

b) correct dominance factor.

On the other hand, different views proposed to explain heterosis focus on three points, which are:

1. partial dominance,

2. super dominance and

3. varied epistasis.

However, the correct thesis has not yet been determined.

Studies on the properties under the influence of the combining ability are especially important for successful hybrid breeding. Combining ability is defined as the ability to transfer the desired properties of appropriated lines entered into hybrid combinations to hybrid offspring (Hayes and Immer, 1942). Sprague and Tatum (1942) define general combining ability as the average performance of a line in a hybrid combination and specific combining ability as the better or poorer performance than expected of a given hybrid combination. Properties under the influence of general combining ability are affected by additive gene action, while properties under the influence of specific combining ability are affected by non-additive gene action or dominant and/or epistatic gene action (Falconer, 1975). Falconer (1975) 
established that the difference in general combining ability stems from additive variance and additive $\times$ additive interaction due to different environmental conditions, while the difference in specific combining ability is attributed to non-additive genetic variance. General/specific combining power can be estimated via various methods, the most common of which is diallel analysis (Griffling, 1956). Line $\times$ tester (multiple sequence) analysis is the modified version of the top-cross method proposed by Kemptorn (1957) and is used as a suitable method for hybrid variety breeding programs where especially cytoplasmic sterile and restorer lines are included as parents (Singh and Chaudary, 1977). The main objective of this study is to calculate general and specific combining ability and heterosis, heterobeltiosis values and heritability to identify hybrids that are suitable in terms of the addressed properties for parent breeding.

\section{MATERIAL AND METHODS}

In this study, five cytoplasmic male sterile (cms) lines ( $\mathrm{cms}$ 0043, $\mathrm{cms}$ 0046, cms 0195, cms 0583 ve cms 0704) and four restorer (Rf) lines (0708 Rf, $0845 R f$, $0951 R f$ ve $1097 R f$ ) were hybridized via the line $\times$ tester (multiple sequence) method. Five cytoplasmic male sterile $(\mathrm{cms})$ and 4 restorer lines were crossed. The plant characters: seed yield (g), 1000-seed weight (g), days to flowering, days to physiological maturity, plant height $(\mathrm{cm})$, head diameter $(\mathrm{cm})$, stem diameter at the bottom internode $(\mathrm{cm})$, stem diameter at stem curve point $(\mathrm{cm})$, oil content \%, oleic acid content \%, linoleic acid content \%, palmitic acid content \%, stearic acid content $\%$, protein content $\%$, seed length $(\mathrm{mm})$, seed width $(\mathrm{mm})$, and hull percentage were studied.

Parental lines (cms and $R f$ ) in the study were as follows:

\begin{tabular}{lccc}
\hline Parents & $c m s / R f$ & Habitus & Type \\
\hline 0043 & $c m s^{*}$ & Non-branched, Single headed & Oilseed \\
0046 & $\mathrm{cms}$ & Non-branched, Single headed & Oilseed \\
0195 & $\mathrm{cms}$ & Non-branched, Single headed & Oilseed \\
0583 & $\mathrm{cms}$ & Non-branched, Single headed & Oilseed \\
0704 & $\mathrm{cms}$ & Non-branched, Single headed & Oilseed \\
0708 & $\mathrm{RfRf}$ & Non-branched, Single headed & Oilseed \\
0845 & $\mathrm{RfRf}$ & Non-branched, Single headed & Oilseed \\
0951 & $\mathrm{RfRf}$ & Non-branched, Single headed & Oilseed \\
1097 & RfRf & Non-branched, Single headed & Oilseed \\
\hline
\end{tabular}

${ }^{\star}$ cms : Cytoplasmic male sterile line; $R f$ : Restorer line

Parents and their hybrids were planted in three rows per plot. The rows were $0.70 \mathrm{~m}$ apart and $7.70 \mathrm{~m}$ in length. At the harvest, the $1^{\text {st }}$ and $3^{\text {rd }}$ rows and first and last plants of the middle row were removed as the edge effect and 20 plants in the middle were harvested for evaluation. 
All values were first evaluated via variance analysis (VA) (Steel ve Torrie, 1980), after which line $\times$ tester analysis was applied (Singh ve Chaudary, 1977).

A two-sided table was created for lines (cms) and testers $(R f)$ in the line $\times$ tester analysis to degrade hybrid squares into the mother, father, and mother $\times$ father interaction components (Singh and Chaudary, 1977).

Hybrid values were created on the basis of average values (Arunachalam, 1974).

GCA effects of lines and testers, SCA effects of lines $\times$ testers, and the variances of these values were calculated (Griffling, 1956; Kempthorn, 1957; Rao et al., 1968; Singh and Chaudary, 1977).

The $\mathrm{KOV}(\mathrm{HS})$ average value was equivalent to the GCA variance and the KOV(FS) value was equivalent to the SCA variance. These values and additive variance $\left(\sigma^{2} \mathrm{D}\right)$ and dominance variance $\left(\sigma^{2} \mathrm{H}\right)$ components were used to identify proportional relations.

General (GCA) and specific combining ability (SCA) and heterosis of inbred lines and their hybrids were estimated in a line $\times$ tester analysis during the first and second crop production seasons in Menemen, Izmir - Turkey.

Heterosis, heterobeltiosis (Hallauer and Eberhard, 1966; Hallauer and Miranda, 1981):

$\%$ Heterosis $(\mathrm{Hs})=\frac{\mathrm{F}_{1}-\mathrm{MP}}{\mathrm{MP}} \times 100$

Significance check of heterosis (Cochran and Cox, 1957; Steel and Torrie, 1980):

$\%$ Heterobeltiosis $(\mathrm{Hb})=\frac{\mathrm{F}_{1}-\mathrm{HP}}{\mathrm{HP}} \times 100$

Significance check of heterobeltiosis was performed via LSD test (Fonseca ve Patterson, 1968).

Broad sense heritability $(\mathrm{H})$ values were calculated according to Kempthorn (1957).

$\mathrm{H}=(\mathrm{G} / \mathrm{F})$ and $\mathrm{h}^{2}=(\mathrm{A} / \mathrm{F})$

Narrow sense heritability $\left(h^{2}\right)$ values were calculated according to Falconer (1975).

Floral receptacles of all $\mathrm{cms}$ and $R f$ lines were isolated before flowering for the purpose of preventing allogamy. Twenty hybrid $\left(\mathrm{F}_{1}\right)$ and nine parent seeds were cultivated according to a randomized block design with four replications under main (primary) and secondary product conditions and evaluated in terms of the addressed properties. The cultivation was in three rows per plot with a length of $7.70 \mathrm{~cm}$ and an interval of $70 \mathrm{~cm}$. During the harvest, one plant each from the $1^{\text {st }}$ and $3^{\text {rd }}$ rows and the middle row of each plot were removed as the edge effect and 20 plants in the middle were harvested for evaluation. The properties addressed in 
the main and secondary product in the trial plots and harvested seeds were plot yield, 1000-kernel weight, days to flowering, days to physiological maturity, plant weight, receptacle diameter, grain size, grain width, hull ratio, oil ratio, protein ratio, oleic acid ratio, linoleic acid ratio, palmitic acid ratio, stearic acid ratio, stem diameter and head diameter. The findings were processed through pre-variance analysis (Steel and Torrie, 1980) and in case of the presence of inter-genotypic variance; line $\times$ tester (multiple sequence) analysis was implemented (Singh and Chaudary, 1977).

Effects of general and specific combining abilities and general and specific combination variances were calculated according to the method proposed by Kempthorn (1957), Rao et al. (1968), and Singh and Chaudary (1977). First, a two-sided table (Singh and Chaudary, 1977) was created for lines (cms) and testers $(R f)$ in the line $\times$ tester analysis and the values thus obtained were used to calculate total repetition values of combinations via degrading hybrid squares into the mother, father, and mother $\times$ father interaction components. Hybrid values were created on the basis of average values (Arunachalam, 1974). Two-sided table values, general combining ability effects of lines and testers, and specific combining ability effects of lines $\times$ testers - via the method proposed by Griffling (1956) - and the standard deviations of these values were calculated. The KOV(HS) average value was equivalent to the general combining ability variance and the $\mathrm{KOV}(\mathrm{FS})$ value to the specific combining ability; these values and additive $(\mathrm{D})$, dominance $(\mathrm{H})$ variance components were used to identify proportional relations. Heterosis (\%) and heterobeltiosis (\%) values for the properties addressed in the study were calculated according to Hallauer and Eberhard (1966) and Hallauer and Miranda (1981). Significance check of heterosis was calculated according to Cochran and Cox (1957) and Steel and Torrie (1980), while the significance check of heterobeltiosis was performed via LSD test (Fonseca and Patterson, 1968). Narrow $\left(\mathrm{h}^{2}\right)$ and broad $(\mathrm{H})$ sense heritability values were calculated according to Kempthorn (1957) and Falconer (1975).

In this study, the statistical significance of the mean square values of the addressed properties confirmed the presence of variance in the research material in terms of these properties.

\section{RESULTS AND DISCUSSION}

Variance estimates for GCA and SCA, dominance and additive variance components, and their proportional relations were determined.

All characters were evaluated via variance analysis and highly significant differences were found among the genotypes (Steel and Torrie, 1980) (Tables 1, 2, 3, 4, 5 , and 6).

Line $\times$ tester analysis (Singh and Chaudary, 1977) indicated that there were significant variations present among the parents and their hybrids for all the traits studied (Tables 7, 8, 9, and 10). 
Table 1: First crop mean values of the characters plot seed yield, days to flowering, days to physiological maturity, plant height $(\mathrm{cm})$, head diameter $(\mathrm{cm})$, and 1000-seed weight.

\begin{tabular}{|c|c|c|c|c|c|c|c|}
\hline Genotypes & No. & $\begin{array}{l}\text { Seed } \\
\text { yield }\end{array}$ & $\begin{array}{l}\text { Days to } \\
\text { flowering }\end{array}$ & $\begin{array}{c}\text { Days to } \\
\text { physiological } \\
\text { maturity }\end{array}$ & $\begin{array}{l}\text { Plant } \\
\text { height }\end{array}$ & $\begin{array}{c}\text { Head } \\
\text { diameter }\end{array}$ & $\begin{array}{c}\text { 1000-seed } \\
\text { weight }\end{array}$ \\
\hline & & $\left(\right.$ g plot $\left.^{-1}\right)$ & (day) & (day) & $(\mathrm{cm})$ & $(\mathrm{cm})$ & (g) \\
\hline $0043 \mathrm{cms}$ & 1 & 1243 & 64.00 & 101.00 & 160.00 & 18.33 & 54.28 \\
\hline $0046 \mathrm{cms}$ & 2 & 1041 & 62.75 & 103.50 & 134.40 & 18.38 & 48.17 \\
\hline $0195 \mathrm{cms}$ & 3 & 969 & 65.25 & 106.50 & 129.10 & 17.15 & 80.22 \\
\hline $0583 \mathrm{cms}$ & 4 & 1038 & 64.25 & 104.00 & 114.90 & 16.05 & 48.26 \\
\hline $0704 \mathrm{cms}$ & 5 & 1155 & 66.00 & 108.30 & 146.70 & 16.63 & 56.55 \\
\hline $0708 R f$ & 6 & 259 & 60.75 & 88.50 & 107.10 & 8.23 & 16.91 \\
\hline $0845 R f$ & 7 & 396 & 67.25 & 101.00 & 116.30 & 9.35 & 24.12 \\
\hline $0951 R f$ & 8 & 766 & 63.25 & 103.50 & 111.70 & 15.20 & 47.12 \\
\hline $1097 R f$ & 9 & 293 & 57.00 & 96.25 & 112.00 & 7.90 & 25.32 \\
\hline $0043 \times 0708$ & 10 & 1480 & 59.50 & 101.30 & 174.30 & 19.20 & 56.45 \\
\hline $0043 \times 0708$ & 11 & 1544 & 63.75 & 104.00 & 178.20 & 19.23 & 54.74 \\
\hline $0043 \times 0951$ & 12 & 1385 & 61.75 & 101.00 & 146.80 & 16.27 & 45.58 \\
\hline $0043 \times 1097$ & 13 & 1399 & 60.50 & 101.00 & 168.90 & 18.63 & 57.95 \\
\hline $0046 \times 0708$ & 14 & 1501 & 61.75 & 101.80 & 169.20 & 19.33 & 53.90 \\
\hline $0046 \times 0845$ & 15 & 1433 & 64.00 & 104.00 & 164.00 & 18.55 & 59.47 \\
\hline $0046 \times 0951$ & 16 & 1436 & 62.25 & 105.80 & 150.80 & 17.60 & 51.92 \\
\hline $0046 \times 1097$ & 17 & 1329 & 61.00 & 104.00 & 165.60 & 17.33 & 53.48 \\
\hline $0195 \times 0708$ & 18 & 1345 & 60.00 & 100.00 & 147.50 & 16.77 & 57.22 \\
\hline $0195 \times 0845$ & 19 & 1328 & 61.75 & 102.80 & 139.30 & 17.55 & 59.81 \\
\hline $0195 \times 0951$ & 20 & 1225 & 61.00 & 99.50 & 122.00 & 15.32 & 51.82 \\
\hline $0195 \times 1097$ & 21 & 1350 & 60.00 & 103.50 & 149.60 & 16.60 & 62.33 \\
\hline $0583 \times 0708$ & 22 & 1423 & 58.50 & 105.30 & 137.70 & 17.77 & 52.16 \\
\hline $0583 \times 0845$ & 23 & 1358 & 61.75 & 102.00 & 140.90 & 17.95 & 51.08 \\
\hline $0583 \times 0951$ & 24 & 1273 & 60.50 & 104.00 & 134.20 & 16.45 & 53.89 \\
\hline $0583 \times 1097$ & 25 & 1366 & 56.50 & 101.30 & 135.10 & 17.95 & 56.86 \\
\hline $0704 \times 0708$ & 26 & 1621 & 62.00 & 103.30 & 150.50 & 17.73 & 47.92 \\
\hline $0704 \times 0845$ & 27 & 1388 & 63.75 & 106.00 & 153.30 & 17.75 & 52.03 \\
\hline $0704 \times 0951$ & 28 & 1343 & 66.25 & 107.30 & 163.50 & 17.65 & 52.79 \\
\hline $0704 \times 1097$ & 29 & 1420 & 60.50 & 106.00 & 164.60 & 18.85 & 62.74 \\
\hline LSD $(\alpha=0.05)$ & & 224.300 & 1.510 & 1.282 & 21.560 & 2.002 & 9.210 \\
\hline $\operatorname{LSD}(\alpha=0.01)$ & & 297.300 & 2.001 & 1.699 & 28.570 & 2.654 & 12.210 \\
\hline
\end{tabular}

* Significant $\alpha=0.01$ 
Table 2: First crop mean values of the characters hull percentage (\%), seed length (mm), seed width $(\mathrm{mm})$, and stem lower part and curve diameters $(\mathrm{mm})$.

\begin{tabular}{|c|c|c|c|c|c|c|}
\hline \multirow[t]{2}{*}{ Genotypes } & \multirow[t]{2}{*}{ No. } & $\begin{array}{c}\text { Hull } \\
\text { percentage }\end{array}$ & $\begin{array}{l}\text { Seed } \\
\text { length }\end{array}$ & $\begin{array}{l}\text { Seed } \\
\text { width }\end{array}$ & $\begin{array}{c}\text { Stem lower } \\
\text { part diameter }\end{array}$ & $\begin{array}{l}\text { Stem curve } \\
\text { part diameter }\end{array}$ \\
\hline & & (\%) & $(\mathrm{mm})$ & $(\mathrm{mm})$ & $(\mathrm{cm})$ & $(\mathrm{cm})$ \\
\hline $0043 \mathrm{cms}$ & 1 & 27.90 & 11.58 & 5.71 & 2.61 & 1.52 \\
\hline $0046 \mathrm{cms}$ & 2 & 25.90 & 10.76 & 5.50 & 2.58 & 1.41 \\
\hline $0195 \mathrm{cms}$ & 3 & 23.93 & 12.64 & 5.83 & 2.49 & 1.83 \\
\hline $0583 \mathrm{cms}$ & 4 & 15.60 & 12.23 & 4.32 & 2.41 & 1.50 \\
\hline $0704 \mathrm{cms}$ & 5 & 20.19 & 11.06 & 5.44 & 2.75 & 1.49 \\
\hline $0708 R f$ & 6 & 28.66 & 9.28 & 3.36 & 2.12 & 0.92 \\
\hline $0845 R f$ & 7 & 20.30 & 9.25 & 4.58 & 2.22 & 1.00 \\
\hline $0951 R f$ & 8 & 25.58 & 12.39 & 5.09 & 2.35 & 1.42 \\
\hline $1097 R f$ & 9 & 20.17 & 8.50 & 4.30 & 1.69 & 1.09 \\
\hline $0043 \times 0708$ & 10 & 24.56 & 10.82 & 5.46 & 2.77 & 1.48 \\
\hline $0043 \times 0708$ & 11 & 24.85 & 11.41 & 5.86 & 2.79 & 1.49 \\
\hline $0043 \times 0951$ & 12 & 24.60 & 11.48 & 5.21 & 2.45 & 1.30 \\
\hline $0043 \times 1097$ & 13 & 25.85 & 10.74 & 5.59 & 2.72 & 1.50 \\
\hline $0046 \times 0708$ & 14 & 24.16 & 10.35 & 5.33 & 2.93 & 1.52 \\
\hline $0046 \times 0845$ & 15 & 23.22 & 11.26 & 6.07 & 2.80 & 1.52 \\
\hline $0046 \times 0951$ & 16 & 22.65 & 11.29 & 5.17 & 2.50 & 1.34 \\
\hline $0046 \times 1097$ & 17 & 22.81 & 10.19 & 5.60 & 2.70 & 1.40 \\
\hline $0195 \times 0708$ & 18 & 23.94 & 11.46 & 4.98 & 2.60 & 1.75 \\
\hline $0195 \times 0845$ & 19 & 21.76 & 12.28 & 5.76 & 2.37 & 1.51 \\
\hline $0195 \times 0951$ & 20 & 24.41 & 12.24 & 5.30 & 2.33 & 1.33 \\
\hline $0195 \times 1097$ & 21 & 22.80 & 11.54 & 5.63 & 2.40 & 1.52 \\
\hline $0583 \times 0708$ & 22 & 21.91 & 11.43 & 4.77 & 2.69 & 1.59 \\
\hline $0583 \times 0845$ & 23 & 17.84 & 11.74 & 5.25 & 2.60 & 1.52 \\
\hline $0583 \times 0951$ & 24 & 19.30 & 12.18 & 5.02 & 2.69 & 1.54 \\
\hline $0583 \times 1097$ & 25 & 20.11 & 11.03 & 5.27 & 2.43 & 1.54 \\
\hline $0704 \times 0708$ & 26 & 25.64 & 10.66 & 4.97 & 2.61 & 1.42 \\
\hline $0704 \times 0845$ & 27 & 21.95 & 11.11 & 5.58 & 2.64 & 1.45 \\
\hline $0704 \times 0951$ & 28 & 22.63 & 11.43 & 4.97 & 2.86 & 1.39 \\
\hline $0704 \times 1097$ & 29 & 23.13 & 10.65 & 5.61 & 2.63 & 1.54 \\
\hline $\operatorname{LSD}(\alpha=0.05)$ & & 2.583 & 0.380 & 0.305 & 0.393 & 0.235 \\
\hline $\operatorname{LSD}(\alpha=0.01)$ : & & 3.424 & 0.504 & 0.404 & 0.521 & 0.312 \\
\hline
\end{tabular}

* Significant $\alpha=0.01$ 
Table 3: First crop mean values of the characters oil content (\%), oleic acid content (\%), linoleic acid content (\%), palmitic acid content (\%), stearic acid content (\%), and protein content (\%).

\begin{tabular}{|c|c|c|c|c|c|c|c|}
\hline \multirow[t]{2}{*}{ Genotypes } & \multirow[t]{2}{*}{ No. } & \multirow{2}{*}{$\begin{array}{c}\begin{array}{c}\text { Oil } \\
\text { content }\end{array} \\
(\%)\end{array}$} & \multirow{2}{*}{$\begin{array}{c}\begin{array}{c}\text { Oleic acid } \\
\text { content }\end{array} \\
(\%)\end{array}$} & \multirow{2}{*}{$\begin{array}{c}\begin{array}{c}\text { Linoleic acid } \\
\text { content }\end{array} \\
(\%)\end{array}$} & \multirow{2}{*}{$\begin{array}{c}\begin{array}{c}\text { Palmitic acid } \\
\text { content }\end{array} \\
(\%)\end{array}$} & \multirow{2}{*}{$\begin{array}{c}\begin{array}{c}\text { Stearic acid } \\
\text { content }\end{array} \\
(\%)\end{array}$} & \multirow{2}{*}{$\begin{array}{c}\begin{array}{c}\text { Protein } \\
\text { content }\end{array} \\
(\%)\end{array}$} \\
\hline & & & & & & & \\
\hline $0043 \mathrm{cms}$ & 1 & 40.60 & 43.38 & 42.57 & 7.68 & 5.53 & 20.76 \\
\hline $0046 \mathrm{cms}$ & 2 & 41.42 & 48.08 & 38.75 & 6.69 & 5.74 & 21.47 \\
\hline $0195 \mathrm{cms}$ & 3 & 43.16 & 44.49 & 43.24 & 6.43 & 5.13 & 20.39 \\
\hline $0583 \mathrm{cms}$ & 4 & 49.06 & 34.39 & 52.16 & 6.91 & 5.52 & 22.77 \\
\hline $0704 \mathrm{cms}$ & 5 & 52.34 & 40.14 & 48.37 & 6.88 & 3.88 & 18.99 \\
\hline $0708 R f$ & 6 & 37.91 & 40.92 & 45.95 & 7.39 & 4.49 & 20.12 \\
\hline $0845 R f$ & 7 & 50.67 & 35.69 & 48.64 & 7.62 & 6.63 & 21.32 \\
\hline $0951 R f$ & 8 & 39.56 & 40.53 & 46.02 & 7.04 & 5.17 & 21.49 \\
\hline $1097 R f$ & 9 & 45.22 & 38.50 & 47.54 & 8.24 & 4.75 & 23.49 \\
\hline $0043 \times 0708$ & 10 & 50.16 & 45.17 & 42.44 & 6.48 & 4.60 & 18.56 \\
\hline $0043 \times 0845$ & 11 & 49.92 & 42.62 & 44.71 & 6.85 & 4.86 & 17.55 \\
\hline $0043 \times 0951$ & 12 & 43.60 & 38.85 & 47.63 & 7.00 & 5.11 & 18.76 \\
\hline $0043 \times 1097$ & 13 & 48.15 & 44.17 & 43.51 & 6.72 & 4.55 & 19.44 \\
\hline $0046 \times 0708$ & 14 & 49.60 & 46.40 & 41.87 & 6.27 & 4.48 & 19.55 \\
\hline $0046 \times 0845$ & 15 & 50.69 & 42.47 & 44.97 & 6.80 & 4.98 & 18.58 \\
\hline $0046 \times 0951$ & 16 & 50.53 & 39.84 & 48.02 & 6.84 & 4.55 & 18.44 \\
\hline $0046 \times 1097$ & 17 & 50.36 & 44.32 & 43.50 & 6.79 & 4.53 & 19.05 \\
\hline $0195 \times 0708$ & 18 & 47.76 & 40.22 & 47.63 & 6.63 & 4.42 & 17.29 \\
\hline $0195 \times 0845$ & 19 & 52.41 & 38.58 & 48.79 & 6.88 & 4.88 & 16.47 \\
\hline $0195 \times 0951$ & 20 & 42.65 & 38.24 & 48.17 & 7.35 & 5.32 & 19.44 \\
\hline $0195 \times 1097$ & 21 & 49.46 & 43.57 & 44.14 & 6.54 & 4.91 & 18.91 \\
\hline $0583 \times 0708$ & 22 & 52.03 & 41.50 & 46.58 & 6.19 & 4.61 & 17.67 \\
\hline $0583 \times 0845$ & 23 & 54.83 & 38.11 & 48.32 & 6.56 & 5.72 & 18.74 \\
\hline $0583 \times 0951$ & 24 & 47.04 & 40.30 & 46.83 & 6.43 & 5.65 & 22.58 \\
\hline $0583 \times 0197$ & 25 & 51.58 & 43.52 & 43.35 & 7.06 & 5.44 & 20.37 \\
\hline $0704 \times 0708$ & 26 & 48.64 & 40.87 & 47.86 & 7.02 & 3.91 & 17.34 \\
\hline $0704 \times 0845$ & 27 & 51.88 & 37.22 & 50.60 & 7.33 & 4.26 & 16.24 \\
\hline $0704 \times 0951$ & 28 & 51.13 & 37.77 & 50.19 & 6.96 & 3.90 & 17.58 \\
\hline $0704 \times 1097$ & 29 & 49.71 & 42.24 & 46.01 & 7.04 & 4.42 & 19.40 \\
\hline $\operatorname{LSD}(\alpha=0.05)$ & & 4.818 & 2.957 & 3.035 & 0.716 & 0.716 & 2.433 \\
\hline $\operatorname{LSD}(\alpha=0.01)$ & & 6.386 & 3.919 & 4.022 & 0.943 & 0.949 & 3.224 \\
\hline
\end{tabular}

* Significant $\alpha=0.01$ 
Table 4: Second crop mean values of the characters plot seed yield, days to flowering, days to physiological maturity, plant height $(\mathrm{cm})$, head diameter $(\mathrm{cm})$, and 1000-seed weight.

\begin{tabular}{lccccccc}
\hline \multirow{2}{*}{ Genotypes } & No & $\begin{array}{c}\text { Seed } \\
\text { yield }\end{array}$ & $\begin{array}{c}\text { Days to } \\
\text { flowering }\end{array}$ & $\begin{array}{c}\text { Days to physio- } \\
\text { logical maturity }\end{array}$ & $\begin{array}{c}\text { Plant } \\
\text { height }\end{array}$ & $\begin{array}{c}\text { Head } \\
\text { diameter }\end{array}$ & $\begin{array}{c}1000-\text { seed } \\
\text { weight }\end{array}$ \\
\cline { 2 - 8 } & & (g plot $\left.^{-1}\right)$ & (day) & (day) & (cm) & $(\mathrm{cm})$ & $(\mathrm{g})$ \\
\hline $0043 \mathrm{cms}$ & 1 & 1040 & 47.25 & 90.25 & 160.90 & 17.60 & 59.04 \\
$0046 \mathrm{cms}$ & 2 & 839 & 46.25 & 91.75 & 162.00 & 19.88 & 56.93 \\
$0195 \mathrm{cms}$ & 3 & 718 & 46.00 & 88.50 & 128.20 & 16.45 & 80.86 \\
$0583 \mathrm{cms}$ & 4 & 829 & 47.00 & 90.50 & 142.40 & 16.68 & 68.51 \\
$0704 \mathrm{cms}$ & 5 & 878 & 49.25 & 91.00 & 155.90 & 17.05 & 53.74 \\
$0708 \mathrm{Rf}$ & 6 & 301 & 43.25 & 78.00 & 107.60 & 9.14 & 14.90 \\
$0845 \mathrm{Rf}$ & 7 & 301 & 47.00 & 88.25 & 114.10 & 9.10 & 18.95 \\
$0951 \mathrm{Rf}$ & 8 & 684 & 47.50 & 89.75 & 132.20 & 15.25 & 47.97 \\
$1097 \mathrm{Rf}$ & 9 & 344 & 43.75 & 88.50 & 128.90 & 7.71 & 21.07 \\
$0043 \times 0708$ & 10 & 1134 & 42.00 & 88.00 & 164.70 & 18.24 & 55.22 \\
$0043 \times 0708$ & 11 & 1074 & 44.25 & 90.00 & 176.90 & 17.30 & 53.95 \\
$0043 \times 0951$ & 12 & 1356 & 44.75 & 89.00 & 177.40 & 17.34 & 52.80 \\
$0043 \times 1097$ & 13 & 1463 & 43.75 & 90.25 & 180.50 & 19.09 & 64.89 \\
$0046 \times 0708$ & 14 & 1269 & 42.75 & 91.00 & 169.60 & 18.80 & 55.10 \\
$0046 \times 0845$ & 15 & 1179 & 45.25 & 90.50 & 175.90 & 18.36 & 58.23 \\
$0046 \times 0951$ & 16 & 1345 & 45.25 & 90.50 & 167.90 & 18.46 & 52.26 \\
$0046 \times 1097$ & 17 & 1366 & 44.50 & 90.50 & 183.10 & 19.09 & 61.28 \\
$0195 \times 0708$ & 18 & 1171 & 42.00 & 86.25 & 147.10 & 17.24 & 51.20 \\
$0195 \times 0845$ & 19 & 1221 & 42.50 & 88.75 & 157.40 & 17.65 & 68.79 \\
$0195 \times 0951$ & 20 & 1180 & 43.25 & 86.50 & 147.60 & 16.71 & 60.62 \\
$0195 \times 1097$ & 21 & 1288 & 42.75 & 90.50 & 165.20 & 17.55 & 72.83 \\
$0583 \times 0708$ & 22 & 1230 & 42.00 & 88.75 & 150.50 & 18.54 & 47.32 \\
$0583 \times 0845$ & 23 & 1176 & 43.25 & 89.00 & 157.10 & 17.43 & 55.25 \\
$0583 \times 0951$ & 24 & 1291 & 44.25 & 89.25 & 157.40 & 17.45 & 52.37 \\
$0583 \times 1097$ & 25 & 1270 & 41.25 & 89.00 & 156.40 & 18.50 & 65.88 \\
$0704 \times 0708$ & 26 & 1205 & 43.25 & 89.25 & 160.80 & 18.56 & 42.89 \\
$0704 \times 0845$ & 27 & 1114 & 46.25 & 91.00 & 167.90 & 18.03 & 52.14 \\
$0704 \times 0951$ & 28 & 1126 & 47.75 & 93.00 & 173.10 & 17.96 & 47.98 \\
$0704 \times 1097$ & 29 & 1378 & 44.25 & 93.25 & 165.80 & 18.39 & 61.87 \\
\hline LSD $(\alpha=0.05):$ & & 186.100 & 1.001 & 0.780 & 18.920 & 1.537 & 7.905 \\
LSD $(\alpha=0.01):$ & & 246.600 & 1.327 & 1.034 & 25.080 & 2.036 & 10.480 \\
\hline$*$ Signifcant $\alpha=01$ & & & & & & &
\end{tabular}

* Significant $\alpha=0.01$ 
Table 5: Second crop mean values of the characters hull percentage (\%), seed length ( $\mathrm{mm})$, seed width $(\mathrm{mm})$, and stem lower part and curve diameter $(\mathrm{mm})$.

\begin{tabular}{|c|c|c|c|c|c|c|}
\hline \multirow[t]{2}{*}{ Genotypes } & \multirow[t]{2}{*}{ No } & $\begin{array}{c}\text { Hull } \\
\text { percentage }\end{array}$ & $\begin{array}{l}\text { Seed } \\
\text { length }\end{array}$ & $\begin{array}{l}\text { Seed } \\
\text { width }\end{array}$ & $\begin{array}{c}\text { Stem lower } \\
\text { part diameter }\end{array}$ & $\begin{array}{c}\text { Stem curve } \\
\text { part diameter }\end{array}$ \\
\hline & & (\%) & $(\mathrm{mm})$ & $(\mathrm{mm})$ & $(\mathrm{cm})$ & $(\mathrm{cm})$ \\
\hline $0043 \mathrm{cms}$ & 1 & 29.39 & 12.15 & 6.04 & 2.38 & 1.32 \\
\hline $0046 \mathrm{cms}$ & 2 & 24.08 & 11.25 & 5.85 & 2.91 & 1.50 \\
\hline $0195 \mathrm{cms}$ & 3 & 23.72 & 13.19 & 5.96 & 2.30 & 1.70 \\
\hline $0583 \mathrm{cms}$ & 4 & 16.30 & 12.98 & 5.18 & 2.37 & 1.46 \\
\hline $0704 \mathrm{cms}$ & 5 & 22.24 & 11.34 & 5.04 & 2.62 & 1.42 \\
\hline $0708 R f$ & 6 & 27.04 & 9.20 & 3.17 & 1.85 & 1.00 \\
\hline $0845 R f$ & 7 & 16.05 & 8.62 & 4.06 & 2.00 & 0.95 \\
\hline $0951 R f$ & 8 & 28.80 & 12.88 & 5.47 & 2.29 & 1.52 \\
\hline $1097 R f$ & 9 & 17.91 & 8.36 & 3.82 & 1.82 & 1.00 \\
\hline $0043 \times 0708$ & 10 & 28.13 & 11.25 & 6.05 & 2.42 & 1.35 \\
\hline $0043 \times 0708$ & 11 & 29.54 & 11.99 & 6.19 & 2.65 & 1.34 \\
\hline $0043 \times 0951$ & 12 & 27.12 & 12.13 & 5.71 & 2.78 & 1.29 \\
\hline $0043 \times 1097$ & 13 & 26.48 & 11.44 & 6.20 & 2.77 & 1.47 \\
\hline $0046 \times 0708$ & 14 & 24.75 & 10.74 & 5.76 & 2.67 & 1.43 \\
\hline $0046 \times 0845$ & 15 & 25.99 & 11.31 & 6.35 & 2.84 & 1.33 \\
\hline $0046 \times 0951$ & 16 & 24.88 & 11.62 & 5.55 & 2.71 & 1.34 \\
\hline $0046 \times 1097$ & 17 & 23.07 & 10.79 & 5.89 & 2.87 & 1.35 \\
\hline $0195 \times 0708$ & 18 & 26.12 & 12.02 & 5.48 & 2.27 & 1.37 \\
\hline $0195 \times 0845$ & 19 & 22.21 & 12.66 & 6.28 & 2.46 & 1.66 \\
\hline $0195 \times 0951$ & 20 & 24.43 & 13.08 & 5.85 & 2.40 & 1.60 \\
\hline $0195 \times 1097$ & 21 & 23.68 & 11.97 & 5.86 & 2.44 & 1.54 \\
\hline $0583 \times 0708$ & 22 & 24.91 & 11.61 & 5.01 & 2.26 & 1.45 \\
\hline $0583 \times 0845$ & 23 & 19.74 & 11.99 & 5.53 & 2.52 & 1.54 \\
\hline $0583 \times 0951$ & 24 & 21.81 & 12.53 & 5.30 & 2.47 & 1.63 \\
\hline $0583 \times 1097$ & 25 & 18.75 & 11.24 & 5.36 & 2.42 & 1.57 \\
\hline $0704 \times 0708$ & 26 & 27.71 & 10.93 & 5.12 & 2.56 & 1.52 \\
\hline $0704 \times 0845$ & 27 & 23.68 & 11.21 & 5.92 & 2.60 & 1.40 \\
\hline $0704 \times 0951$ & 28 & 24.94 & 11.66 & 5.23 & 2.81 & 1.55 \\
\hline $0704 \times 1097$ & 29 & 23.24 & 11.01 & 5.82 & 2.60 & 1.60 \\
\hline $\operatorname{LSD}(\alpha=0.05)$ & & 2.351 & 0.388 & 0.375 & 0.333 & 0.196 \\
\hline $\operatorname{LSD}(\alpha=0.01)$ & & 3.116 & 0.514 & 0.497 & 0.441 & 0.257 \\
\hline
\end{tabular}

* Significant $\alpha=0.01$ 
Table 6: Second crop mean values of the characters oil content (\%), oleic acid content (\%), linoleic acid content (\%), palmitic acid content (\%), and stearic acid content (\%), and protein content (\%).

\begin{tabular}{lccccccc}
\hline \multirow{2}{*}{ Genotypes } & No & Oil & Oleic acid & Linoleic acid & Palmitic acid & Stearic acid & Protein \\
& & content & content & content & content & content \\
\hline $0043 \mathrm{cms}$ & 1 & 37.56 & 22.02 & 61.94 & 7.83 & 6.85 & 22.62 \\
$0046 \mathrm{cms}$ & 2 & 39.11 & 21.28 & 62.27 & 7.27 & 7.31 & 24.05 \\
$0195 \mathrm{cms}$ & 3 & 38.06 & 24.01 & 58.57 & 7.09 & 7.63 & 24.69 \\
$0583 \mathrm{cms}$ & 4 & 42.25 & 20.28 & 62.13 & 6.89 & 7.57 & 27.65 \\
$0704 \mathrm{cms}$ & 5 & 38.56 & 19.80 & 66.53 & 7.37 & 5.73 & 22.97 \\
$0708 \mathrm{Rf}$ & 6 & 32.35 & 18.40 & 65.58 & 9.26 & 5.80 & 23.38 \\
$0845 \mathrm{Rf}$ & 7 & 42.31 & 17.62 & 67.13 & 8.25 & 6.49 & 26.07 \\
$0951 \mathrm{Rf}$ & 8 & 31.60 & 19.39 & 65.04 & 7.88 & 5.80 & 25.84 \\
$1097 \mathrm{Rf}$ & 9 & 42.88 & 18.78 & 64.08 & 9.37 & 6.81 & 25.60 \\
$0043 \times 0708$ & 10 & 38.06 & 23.46 & 60.88 & 7.31 & 6.32 & 21.89 \\
$0043 \times 0845$ & 11 & 37.45 & 21.42 & 62.32 & 7.71 & 7.43 & 23.07 \\
$0043 \times 0951$ & 12 & 35.72 & 21.68 & 63.24 & 7.18 & 6.93 & 22.17 \\
$0043 \times 1097$ & 13 & 40.97 & 23.42 & 61.65 & 7.06 & 6.68 & 22.59 \\
$0046 \times 0708$ & 14 & 38.33 & 22.73 & 63.21 & 6.78 & 6.45 & 23.19 \\
$0046 \times 0845$ & 15 & 40.04 & 20.85 & 64.79 & 7.13 & 6.65 & 22.46 \\
$0046 \times 0951$ & 16 & 38.19 & 21.12 & 64.24 & 6.77 & 6.55 & 23.58 \\
$0046 \times 1097$ & 17 & 41.90 & 22.36 & 63.03 & 6.80 & 6.57 & 22.27 \\
$0195 \times 0708$ & 18 & 38.95 & 20.52 & 64.54 & 7.36 & 6.46 & 21.43 \\
$0195 \times 0845$ & 19 & 42.83 & 23.17 & 62.19 & 6.76 & 7.01 & 22.82 \\
$0195 \times 0951$ & 20 & 37.36 & 21.77 & 62.96 & 6.79 & 6.98 & 22.93 \\
$0195 \times 1097$ & 21 & 43.30 & 24.45 & 60.64 & 6.42 & 7.10 & 22.98 \\
$0583 \times 0708$ & 22 & 41.56 & 21.72 & 64.13 & 6.43 & 6.36 & 20.85 \\
$0583 \times 0845$ & 23 & 41.99 & 21.36 & 63.62 & 6.65 & 7.51 & 23.23 \\
$0583 \times 0951$ & 24 & 39.05 & 19.35 & 65.70 & 6.80 & 7.13 & 24.67 \\
$0583 \times 0197$ & 25 & 44.81 & 24.12 & 61.09 & 6.34 & 7.02 & 23.46 \\
$0704 \times 0708$ & 26 & 38.06 & 20.74 & 64.91 & 7.39 & 5.94 & 20.77 \\
$0704 \times 0845$ & 27 & 41.08 & 19.46 & 66.48 & 7.47 & 6.01 & 21.46 \\
$0704 \times 0951$ & 28 & 37.96 & 18.59 & 67.41 & 7.46 & 5.70 & 22.15 \\
$0704 \times 1097$ & 29 & 43.35 & 22.27 & 64.00 & 7.19 & 5.91 & 23.16 \\
\hline LSD $(\alpha=0.05):$ & & 4.592 & 1.635 & 2.366 & 0.816 & 0.702 & 2.230 \\
LSD $(\alpha=0.01):$ & & 6.086 & 2.955 & 3.135 & 1.080 & 0.930 & 2.955 \\
\hline$\star S i g n i c a n t \alpha=0.019$ \\
& & & & & & &
\end{tabular}

* Significant $\alpha=0.01$ 
Table 7: Line $\times$ tester variance analysis for the characters plot seed yield, 1000-seed weight, days to flowering, and days to physiological maturity.

\begin{tabular}{|c|c|c|c|c|c|c|}
\hline \multirow{2}{*}{$\begin{array}{l}\text { Source of } \\
\text { variance }\end{array}$} & \multirow{2}{*}{$\begin{array}{l}\text { Crop } \\
\text { production } \\
\text { time }\end{array}$} & \multirow[t]{2}{*}{ Df } & \multirow[t]{2}{*}{$\begin{array}{l}\text { Plot seed } \\
\text { yield }\end{array}$} & \multirow[t]{2}{*}{$\begin{array}{c}1000-\text { seed } \\
\text { weight }\end{array}$} & \multirow{2}{*}{$\begin{array}{c}\begin{array}{c}\text { Days to } \\
\text { flowering }\end{array} \\
\text { (day) }\end{array}$} & \multirow{2}{*}{$\begin{array}{c}\begin{array}{c}\text { Days to physiolo- } \\
\text { gical maturity }\end{array} \\
\text { (day) }\end{array}$} \\
\hline & & & & & & \\
\hline \multirow{2}{*}{ Block } & $\mathrm{M}-1$ & 3 & 28471.55 & 81.01 & $3.23^{\star}$ & 0.57 \\
\hline & M-2 & 3 & $420839.00^{\star *}$ & $750.17^{\star \star}$ & 0.47 & 0.79 \\
\hline \multirow{2}{*}{ Genotype } & $M-1$ & 28 & $512262.60^{\star *}$ & $587.75^{\star \star}$ & $25.55^{\star *}$ & $55.99^{\star *}$ \\
\hline & M-2 & 28 & $417303.00^{\star *}$ & $868.10^{\star *}$ & $16.95^{\star *}$ & $26.06^{\star \star}$ \\
\hline \multirow{2}{*}{ Crosses } & $M-1$ & 19 & 33422.32 & $80.39^{*}$ & $18.01^{* *}$ & $19.03^{\star \star}$ \\
\hline & $M-2$ & 19 & $42329.69^{\star *}$ & $230.98^{\star \star}$ & $10.28^{\star *}$ & $12.32^{\star \star}$ \\
\hline \multirow{2}{*}{ Line } & $M-1$ & 4 & $59262.00^{*}$ & 51.88 & $34.20^{* *}$ & $45.50^{\star *}$ \\
\hline & M-2 & 4 & 18152.00 & $306.54^{* *}$ & $22.04^{\star *}$ & $32.36^{\star *}$ \\
\hline \multirow{2}{*}{ Tester } & $M-1$ & 3 & $72474.66^{*}$ & $201.21^{\star *}$ & $49.63^{\star *}$ & $8.04^{* *}$ \\
\hline & M-2 & 3 & $147773.30^{\star *}$ & $855.71^{* *}$ & $26.78^{\star *}$ & $14.17^{\star *}$ \\
\hline \multirow{2}{*}{ Line $x$ tester } & $M-1$ & 12 & 14916.00 & 59.68 & $4.71^{* *}$ & $12.96^{\star *}$ \\
\hline & M-2 & 12 & 24028.00 & 49.62 & $2.23^{\star *}$ & $5.18^{* *}$ \\
\hline \multirow{2}{*}{ Error } & $\mathrm{M}-1$ & 84 & 25442.38 & 42.90 & 1.15 & 0.83 \\
\hline & M-2 & 84 & 17510.65 & 31.60 & 0.51 & 0.31 \\
\hline
\end{tabular}

* Significant $\alpha=0.05$; ** Significant $\alpha=0.01$; M1: first crop, M2: second crop

Table 8: Line $\times$ tester variance analysis for the characters plant height $(\mathrm{cm})$, head diameter $(\mathrm{cm})$, and stem lower part and curve diameters $(\mathrm{mm})$.

\begin{tabular}{|c|c|c|c|c|c|c|}
\hline \multirow{2}{*}{$\begin{array}{l}\text { Source of } \\
\text { variance }\end{array}$} & \multirow{2}{*}{$\begin{array}{l}\text { Crop } \\
\text { production } \\
\text { time }\end{array}$} & \multirow[t]{2}{*}{ Df } & Plant height & $\begin{array}{c}\text { Head } \\
\text { diameter }\end{array}$ & $\begin{array}{c}\text { Stem lower } \\
\text { part diameter }\end{array}$ & $\begin{array}{l}\text { Stem curve } \\
\text { diameter }\end{array}$ \\
\hline & & & (cm) & (cm) & $(\mathrm{mm})$ & $(\mathrm{mm})$ \\
\hline \multirow{2}{*}{ Block } & $\mathrm{M}-1$ & 3 & 249.52 & $10.93^{\text {** }}$ & $0.26^{*}$ & $0.08^{*}$ \\
\hline & M-2 & 3 & $4822.78^{\star *}$ & $91.04^{* *}$ & $1.92^{\star *}$ & $0.35^{\star *}$ \\
\hline \multirow{2}{*}{ Genotype } & $M-1$ & 28 & $1653.74^{\star *}$ & $36.29^{* *}$ & $0.26^{\star \star}$ & $0.14^{\star \star}$ \\
\hline & M-2 & 28 & $1466.79^{* *}$ & $36.01^{* *}$ & $0.31^{* *}$ & $0.14^{\star *}$ \\
\hline \multirow{2}{*}{ Crosses } & $M-1$ & 19 & $929.77^{\star \star}$ & $4.57^{\star \star}$ & 0.11 & $0.05^{*}$ \\
\hline & M-2 & 19 & $460.55^{\star *}$ & 1.78 & $0.14^{\star \star}$ & $0.05^{\star *}$ \\
\hline \multirow{2}{*}{ Line } & $M-1$ & 4 & $2983.22^{\star *}$ & $8.22^{\star \star}$ & $0.23^{*}$ & 0.04 \\
\hline & M-2 & 4 & $1565.38^{\star *}$ & $4.03^{*}$ & $0.43^{* *}$ & $0.15^{\star \star}$ \\
\hline \multirow{2}{*}{ Tester } & $M-1$ & 3 & $784.33^{\star}$ & $10.50^{* *}$ & 0.10 & $0.17^{* *}$ \\
\hline & M-2 & 3 & $486.33^{*}$ & $3.74^{*}$ & $0.18^{*}$ & 0.03 \\
\hline \multirow{2}{*}{ Line $x$ tester } & $M-1$ & 12 & 281.65 & 1.87 & 0.08 & 0.02 \\
\hline & M-2 & 12 & 85.83 & 0.54 & 0.03 & 0.03 \\
\hline \multirow{2}{*}{ Error } & $\mathrm{M}-1$ & 84 & 234.98 & 2.03 & 0.09 & 0.03 \\
\hline & M-2 & 84 & 181.04 & 1.19 & 0.06 & 0.02 \\
\hline
\end{tabular}

* Significant $\alpha=0.05$; ** Significant $\alpha=0.01$; M1: first crop, M2: second crop 
Table 9: Line $\times$ tester variance analysis for the characters seed length $(\mathrm{mm})$, seed width $(\mathrm{mm})$, hull percentage (\%), and protein content (\%).

\begin{tabular}{|c|c|c|c|c|c|c|}
\hline \multirow{2}{*}{$\begin{array}{l}\text { Source of } \\
\text { variance }\end{array}$} & \multirow{2}{*}{$\begin{array}{l}\text { Crop } \\
\text { production } \\
\text { time }\end{array}$} & \multirow[t]{2}{*}{ Df } & $\begin{array}{l}\text { Seed } \\
\text { length }\end{array}$ & $\begin{array}{l}\text { Seed } \\
\text { width }\end{array}$ & $\begin{array}{c}\text { Hull } \\
\text { percentage }\end{array}$ & $\begin{array}{l}\text { Protein } \\
\text { content }\end{array}$ \\
\hline & & & $(\mathrm{mm})$ & $(\mathrm{mm})$ & (\%) & $(\%)$ \\
\hline \multirow{2}{*}{ Block } & $\mathrm{M}-1$ & 3 & 0.14 & $0.27^{\star \star}$ & $11.24^{*}$ & $3.02^{*}$ \\
\hline & M-2 & 3 & $0.44^{\star *}$ & $0.24^{*}$ & $89.78^{\star *}$ & 5.37 \\
\hline \multirow{2}{*}{ Genotype } & $M-1$ & 28 & $3.67^{\star \star}$ & $1.28^{\star \star}$ & $34.04^{\star *}$ & $13.54^{\star *}$ \\
\hline & M-2 & 28 & $5.61^{\star *}$ & $2.17^{\star \star}$ & $52.67^{* *}$ & $10.03^{* *}$ \\
\hline \multirow{2}{*}{ Crosses } & $M-1$ & 19 & $1.29^{\star *}$ & $0.47^{\star *}$ & $18.60^{* *}$ & $8.06^{\star \star}$ \\
\hline & M-2 & 19 & $1.64^{\star *}$ & $0.61^{\star \star}$ & $28.92^{* *}$ & 3.70 \\
\hline \multirow{2}{*}{ line } & $M-1$ & 4 & $3.09^{* *}$ & $0.60^{* *}$ & $59.38^{* *}$ & $11.55^{* *}$ \\
\hline & M-2 & 4 & $4.31^{* *}$ & $1.46^{\star *}$ & $86.80^{* *}$ & 3.22 \\
\hline \multirow{2}{*}{ Tester } & $M-1$ & 3 & $3.75^{\star *}$ & $1.79^{\star *}$ & $22.10^{* *}$ & $17.50^{\star *}$ \\
\hline & M-2 & 3 & $4.27^{* *}$ & $1.44^{* *}$ & $36.24^{* *}$ & 8.54 \\
\hline \multirow{2}{*}{ linextester } & $M-1$ & 12 & 0.08 & 0.09 & 4.13 & 4.54 \\
\hline & M-2 & 12 & 0.09 & 0.13 & $7.80^{\star *}$ & 2.65 \\
\hline \multirow{2}{*}{ Error } & $\mathrm{M}-1$ & 84 & 0.07 & 0.05 & 3.38 & 2.99 \\
\hline & M-2 & 84 & 0.08 & 0.08 & 2.80 & 2.51 \\
\hline
\end{tabular}

* Significant $\alpha=0.05$; ** Significant $\alpha=0.01$; M1: first crop, M2: second crop

Table 10: Line $\times$ tester variance analysis for the characters; oil percentage (\%), oleic acid (\%), linoleic acid (\%), palmitic acid (\%), and stearic acid (\%).

\begin{tabular}{|c|c|c|c|c|c|c|c|}
\hline \multirow{2}{*}{$\begin{array}{l}\text { Source of } \\
\text { Variance }\end{array}$} & \multirow{2}{*}{$\begin{array}{l}\text { Crop } \\
\text { Production } \\
\text { time }\end{array}$} & \multirow[t]{2}{*}{ Df } & Oil & $\begin{array}{l}\text { Oleic } \\
\text { acid }\end{array}$ & $\begin{array}{l}\text { Linoleic } \\
\text { acid }\end{array}$ & $\begin{array}{l}\text { Palmitic } \\
\text { acid }\end{array}$ & $\begin{array}{c}\text { Stearic } \\
\text { acid }\end{array}$ \\
\hline & & & (\%) & (\%) & (\% & (\%) & (\%) \\
\hline \multirow{2}{*}{ Block } & $\mathrm{M}-1$ & 3 & 11.21 & 10.93 & $21.53^{* *}$ & 0.30 & 0.30 \\
\hline & M-2 & 3 & $89.93^{\star \star}$ & $5.27^{\star *}$ & 4.51 & $5.80^{* *}$ & $1.82^{* *}$ \\
\hline \multirow{2}{*}{ Genotype } & $M-1$ & 28 & $76.86^{\star \star}$ & $41.07^{\star *}$ & $35.85^{\star \star}$ & $0.80^{\star \star}$ & $1.60^{\star *}$ \\
\hline & M-2 & 28 & $38.59^{\star *}$ & $13.41^{* *}$ & $17.53^{* *}$ & $2.09^{* *}$ & $1.36^{* *}$ \\
\hline \multirow{2}{*}{ Crosses } & $M-1$ & 19 & $32.18^{\star \star}$ & $29.25^{\star *}$ & $26.38^{\star \star}$ & 0.40 & $1.04^{* *}$ \\
\hline & M-2 & 19 & $24.61^{* *}$ & $9.93^{\star *}$ & $13.25^{\star \star}$ & 0.60 & $1.03^{\star *}$ \\
\hline \multirow{2}{*}{ Line } & $M-1$ & 4 & $36.73^{*}$ & $41.91^{* *}$ & $49.50^{\star \star}$ & $0.64^{*}$ & $3.18^{\star *}$ \\
\hline & M-2 & 4 & $30.96^{*}$ & $13.23^{\text {** }}$ & $31.91^{* *}$ & $1.89^{\star \star}$ & $3.22^{\star *}$ \\
\hline \multirow{2}{*}{ Tester } & $M-1$ & 3 & $82.60^{\star *}$ & $100.03^{\star *}$ & $71.94^{\star *}$ & 0.66 & $1.20^{\star \star}$ \\
\hline & M-2 & 3 & $101.26^{* *}$ & $28.56^{\star \star}$ & $24.00^{* *}$ & 0.54 & $1.28^{\star \star}$ \\
\hline \multirow{2}{*}{ linextester } & $M-1$ & 12 & 18.06 & 7.34 & 7.28 & 0.25 & 0.29 \\
\hline & M-2 & 12 & 3.32 & $4.18^{\star \star}$ & 4.33 & 0.18 & 0.24 \\
\hline \multirow{2}{*}{ Error } & $\mathrm{M}-1$ & 84 & 11.74 & 4.43 & 4.66 & 0.26 & 0.26 \\
\hline & M-2 & 84 & 10.66 & 1.35 & 2.83 & 0.34 & 0.25 \\
\hline
\end{tabular}

* Significant $\alpha=0.05$; ** Significant $\alpha=0.01$; M1: first crop, M2: second crop. 
The variances due to GCA and SCA were highly significant for most of the characters in both environments (Table 11 and 12). The ratio (H/D) 1/2 and GCA/ SCA suggested that additive gene action was significant for plant height, head diameter, seed length, palmitic acid ratio, stearic acid ratio, stem diameter bottom, and bottom 2. - 3. node point.

Table 11: GCA and SCA variances, additive and dominance variance components, and their relations for hybrids.

\begin{tabular}{lccccccc}
\hline Character & Location & GCA & SCA & GCA/SCA & D & $H$ & (H/D)1/2 \\
\hline Seed yield & $\mathrm{M}-1$ & 16.877 & -109.582 & - & 33.755 & -109.582 & - \\
(g plot $^{-1}$ ) & $\mathrm{M}-2$ & 16.686 & 67.819 & 0.246 & 33.373 & 67.819 & 1.426 \\
1000-seed & $\mathrm{M}-1$ & 0.453 & 4.196 & 0.108 & 0.906 & 4.196 & 2.152 \\
(g) & $\mathrm{M}-2$ & 3.970 & 4.504 & 0.881 & 7.940 & 4.504 & 0.753 \\
Flowering & $\mathrm{M}-1$ & 0.291 & 0.889 & 0.327 & 0.582 & 0.889 & 1.236 \\
(day) & $\mathrm{M}-2$ & 0.176 & 0.430 & 0.409 & 0.352 & 0.430 & 1.105 \\
Physiological & $\mathrm{M}-1$ & 0.133 & 3.032 & 0.044 & 0.266 & 3.032 & 3.376 \\
maturity (day) & $\mathrm{M}-2$ & 0.305 & 1.219 & 0.250 & 0.610 & 1.219 & 1.414 \\
Plant height & $\mathrm{M}-1$ & 14.187 & 11.667 & 1.216 & 28.374 & 11.667 & 0.641 \\
(cm) & $\mathrm{M}-2$ & 8.202 & -23.802 & - & 16.405 & -23.802 & - \\
Head diameter & $\mathrm{M}-1$ & 0.059 & -0.040 & - & 0.118 & -0.040 & - \\
(cm) & $\mathrm{M}-2$ & 0.027 & -0.163 & - & 0.054 & -0.163 & - \\
Stem lower part & $\mathrm{M}-1$ & 0.001 & 0.000 & - & 0.002 & 0.000 & - \\
diameter (mm) & $\mathrm{M}-2$ & 0.002 & -0.007 & - & 0.005 & -0.007 & - \\
Stem curve & $\mathrm{M}-1$ & 0.001 & -0.002 & - & 0.001 & -0.002 & - \\
diameter (mm) & $\mathrm{M}-2$ & 0.001 & 0.003 & 0.182 & 0.001 & 0.003 & 1.634 \\
\hline
\end{tabular}

M1: first crop, M2: second crop

Table 12: GCA and SCA variances, additive and dominance variance components, and their relations for hybrids.

\begin{tabular}{lccccccc}
\hline Character & Location & GCA & SCA & GCA/SCA & D & $H$ & (H/D)1/2 \\
\hline Seed length (mm) & $\mathrm{M}-1$ & 0.027 & 0.001 & 27.000 & 0.053 & 0.001 & 0.137 \\
& $\mathrm{M}-2$ & 0.034 & 0.003 & 11.333 & 0.068 & 0.003 & 0.210 \\
Seed width (mm) & $\mathrm{M}-1$ & 0.008 & 0.011 & 0.727 & 0.016 & 0.011 & 0.829 \\
& $\mathrm{M}-2$ & 0.011 & 0.014 & 0.793 & 0.021 & 0.014 & 0.794 \\
Hull (\%) & $\mathrm{M}-1$ & 0.317 & 0.190 & 1.668 & 0.633 & 0.190 & 0.548 \\
& $\mathrm{M}-2$ & 0.462 & 1.252 & 0.513 & 0.925 & 1.252 & 1.163 \\
Protein (\%) & $\mathrm{M}-1$ & 0.077 & 0.387 & 0.199 & 0.154 & 0.387 & 1.585 \\
& $\mathrm{M}-2$ & 0.023 & 0.035 & 0.657 & 0.046 & 0.035 & 0.872 \\
Oil (\%) & $\mathrm{M}-1$ & 0.309 & 1.580 & 0.196 & 0.618 & 1.580 & 1.599 \\
& $\mathrm{M}-2$ & 0.466 & -1.835 & & 0.932 & -1.835 & \\
Oleic acid (\%) & $\mathrm{M}-1$ & 0.480 & 0.727 & 0.660 & 0.960 & 0.727 & 0.870 \\
\multirow{4}{*}{ Linoleic acid (\%) } & $\mathrm{M}-2$ & 0.126 & 0.706 & 0.178 & 0.252 & 0.706 & 1.674 \\
& $\mathrm{M}-1$ & 0.418 & 0.657 & 0.636 & 0.836 & 0.657 & 0.885 \\
Palmitic acid (\%) & $\mathrm{M}-2$ & 0.195 & 0.376 & 0.519 & 0.390 & 0.376 & 0.982 \\
& $\mathrm{M}-1$ & 0.003 & -0.002 & - & 0.006 & -0.002 & - \\
Stearic acid (\%) & $\mathrm{M}-2$ & 0.009 & -0.038 & - & 0.018 & -0.038 & - \\
& $\mathrm{M}-1$ & 0.016 & 0.007 & 2.730 & 0.003 & 0.007 & 0.452 \\
& $\mathrm{M}-2$ & 0.017 & -0.004 & - & 0.035 & -0.004 & - \\
\hline
\end{tabular}

M1: first crop, M2: second crop 
Dominance (non-additive) gene action was significant for days to flowering, days to physiological maturity, seed width, protein ratio, oleic acid, and linoleic acid ratio. Both types of gene action were significant at different cultivation times: GCA and SCA effects were variable in different seasons; therefore, both types of gene action were observed for seed yield, hull percentage, 1000-seed weight, oil content, and stem diameter at stem curve point. Properties under the dominant gene action demonstrated an $(\mathrm{H} / \mathrm{D}) 1 / 2$ ratio of more than 1 , which indicated the presence of super dominance for these properties.

As a result genetic analysis in different seasons will give better understanding of gene expression before embarking on selection.

In the first and second crop production seasons, the inbreds $0043 \mathrm{cms}, 0046$ cms, $0195 \mathrm{cms}, 0583 \mathrm{cms}, 0704 \mathrm{cms}, 0708 \mathrm{Rf}, 0845 \mathrm{Rf}, 0951 \mathrm{Rf}$, and $1097 \mathrm{Rf}$ exhibited desirable GCA effects and were found to be good general combiners for most of the traits; thus they can be exploited by further breeding for developing superior genotypes and hybrids in sunflower.

When the general combining ability (GCA) values of the parents are concerned, $0195 \mathrm{cms}, 0583 \mathrm{cms}, 0704 \mathrm{cms}, 0708 \mathrm{Rf}$ and $0845 \mathrm{Rf}$ were identified as lines with high general combination ability. These lines can be recommended to be used as parents in different hybrid combinations.

When the specific combining ability of the hybrids is concerned, some combinations can be recommended as hybrids for the first and second crop production times with statistical significance in SCA in terms of the characters studied.

Table 13: Maximum and minimum heterosis and heterobeltiosis values under first crop production time.

\begin{tabular}{|c|c|c|c|c|}
\hline \multirow{3}{*}{ Characters } & \multicolumn{4}{|c|}{ First crop } \\
\hline & \multicolumn{2}{|c|}{ Heterosis (\%) } & \multicolumn{2}{|c|}{ Heterobeltiosis (\%) } \\
\hline & min. & $\max$. & $\min$. & $\max$. \\
\hline Seed yield (g) & 37.90 & 130.96 & 11.47 & 44.18 \\
\hline 1000-seed weight $(\mathrm{g})$ & -18.61 & 65.64 & -35.40 & 17.82 \\
\hline Days to flowering (day) & -6.80 & 2.51 & -12.06 & 0.38 \\
\hline Days to physiological maturity (day) & -5.24 & 9.35 & -6.57 & 2.97 \\
\hline Plant height $(\mathrm{cm})$ & 1.38 & 40.14 & -8.24 & 25.92 \\
\hline Head diameter $(\mathrm{cm})$ & -5.22 & 53.69 & -11.18 & 13.55 \\
\hline Oil content (\%) & 0.71 & 28.16 & -7.05 & 23.61 \\
\hline Oleic acid (\%) & -10.09 & 19.41 & -17.14 & 13.04 \\
\hline Linoleic acid (\%) & -13.04 & 13.29 & -16.89 & 4.69 \\
\hline Palmitic acid (\%) & -15.58 & 10.46 & -20.63 & 10.81 \\
\hline Stearic acid (\%) & -20.07 & 5.94 & -35.75 & 2.90 \\
\hline Protein content (\%) & -21.06 & 2.03 & -23.83 & -0.83 \\
\hline Hull percentage (\%) & -13.15 & 14.62 & -24.55 & 26.99 \\
\hline Seed length $(\mathrm{mm})$ & -4.21 & 12.54 & -9.81 & 4.65 \\
\hline Seed width $(\mathrm{mm})$ & -5.60 & 24.22 & -14.58 & 21.99 \\
\hline Stem diameter (at the bottom, $\mathrm{cm}$ ) & -3.72 & 26.39 & -6.43 & 13.79 \\
\hline Stem diameter (stem curve point, $\mathrm{cm}$ ) & -18.19 & 31.43 & -27.38 & 7.94 \\
\hline
\end{tabular}


The highest level of heterosis (\%) was identified as $142.64 \%$ for seed yield in the hybrid No.21 (195 cms $\times 1097 \mathrm{Rf}$ ), The lowest heterosis level was observed in the palmitic acid ratio with $-22.02 \%$ in the hybrid No. $25(0583 \mathrm{cms} \times 1097 \mathrm{Rf})$.

The highest heterobeltiosis value was observed in the plot yield with $79.44 \%$ (hybrid No.21). The lowest value was observed in 1000-kernel weight with $-36.68 \%$ (hybrid No.18) in the second production time (Tables 13 and 14).

Table 14: Maximum and minimum heterosis and heterobeltiosis values under second crop production time.

\begin{tabular}{|c|c|c|c|c|}
\hline \multirow{3}{*}{ Characters } & \multicolumn{4}{|c|}{ Second crop } \\
\hline & \multicolumn{2}{|c|}{ Heterosis (\%) } & \multicolumn{2}{|c|}{ Heterobeltiosis (\%) } \\
\hline & $\min$. & $\max$. & $\min$. & max. \\
\hline Seed yield (g) & 44.28 & 142.64 & 3.25 & 79.44 \\
\hline 1000-seed weight (g) & -10.08 & 65.41 & -36.68 & 15.13 \\
\hline Days to flowering (day) & -9.09 & -1.11 & -12.23 & -3.05 \\
\hline Days to physiological maturity (day) & -2.95 & 7.22 & -3.62 & 2.47 \\
\hline Plant height $(\mathrm{cm})$ & 13.35 & 29.94 & 2.34 & 28.20 \\
\hline Head diameter $(\mathrm{cm})$ & 5.10 & 51.70 & -7.65 & 11.15 \\
\hline Oil content (\%) & -6.22 & 11.42 & -11.49 & 13.79 \\
\hline Oleic acid (\%) & -5.13 & 23.50 & -14.49 & 18.93 \\
\hline Linoleic acid (\%) & -4.60 & 3.89 & -7.36 & 1.32 \\
\hline Palmitic acid (\%) & -22.02 & -2.16 & -32.34 & -5.33 \\
\hline Stearic acid (\%) & -6.94 & 11.39 & -15.98 & 8.47 \\
\hline Protein content (\%) & -18.28 & -2.21 & -24.59 & -3.58 \\
\hline Hull percentage (\%) & -6.95 & 30.02 & -24.27 & 21.10 \\
\hline Seed length $(\mathrm{mm})$ & -11.02 & 16.19 & -13.41 & 0.83 \\
\hline Seed width $(\mathrm{mm})$ & -1.94 & 31.38 & -8.05 & 17.46 \\
\hline Stem diameter (at the bottom, cm) & 4.23 & 32.16 & -8.25 & 16.71 \\
\hline Stem diameter (stem curve point, $\mathrm{cm}$ ) & -11.26 & 32.23 & -19.41 & 12.68 \\
\hline
\end{tabular}

The highest and lowest narrow sense heritability $\left(\mathrm{h}^{2}\right)$ values in the main production time were 0.810 and 0.059 for seed size and 1000 -seed weight, respectively. In the second production time, the highest and lowest values were 0.756 and 0.065 for seed size and protein ratio, respectively.

The highest and lowest broad sense heritability $(\mathrm{H})$ values in the main production time were 0.990 and 0.239 for seed size and seed yield, respectively, while in the second production time they were 0.951 and 0.330 for days to flowering and head diameter, respectively.

As for variance estimates for general and specific combining ability, dominance and additive variance components, and their proportional relations, additive gene action was significant for plant height, receptacle diameter, grain size, palmitic acid ratio, stearic acid ratio and stem diameter, while non-additive gene action was significant for days to flowering, days to physiological maturity, grain width, protein ratio, oleic acid, and linoleic acid ratio. In addition, different gene actions were significant at different cultivation times for grain yield, 1000-kernel weight, oil ratio, and hull ratio, and head diameter. Accordingly, the non-additive gene effect was sig- 
nificant for the main product and additive gene action for the secondary product for 1000-kernel weight and oil ratio. Additive gene action was significant in the main product and non-additive gene action for the secondary product for head diameter. Properties under the dominant gene action demonstrated an (H/D) 1/2 ratio of more than 1 , which indicated the presence of super dominance for these properties.

When general combining ability values of the parents are concerned, $0195 \mathrm{cms}$, $0583 \mathrm{cms}, 0704 \mathrm{cms}, 0708 \mathrm{Rf}$ and $0845 \mathrm{Rf}$ were identified as lines with high general combination ability in terms of the stearic acid ratio of products. These lines can be recommended to be used as parents in hybrid combinations.

When specific combining ability of hybrids is concerned, hybrid No. 18 (0195) $\times 0708$ ) can be recommended as hybrids with statistical significance in specific combining ability in terms of the linoleic acid ratio of secondary products.

The highest level of heterosis (\%) was identified as $142.64 \%$ for plot yield in the hybrid No. 21, whereas the lowest heterosis level was observed in the palmitic acid ratio with $-22.02 \%$ in the hybrid No. 25 .

As for heterobeltiosis, the highest value was observed for plot yield with $79.44 \%$ (hybrid No.21), while the lowest value was observed for 1000-kernel weight with $36.68 \%$ (hybrid No. 18) in the secondary product.

The highest and lowest values of heritability in the narrow sense were 0.810 (in grain size) and 0.059 (in 1000-kernel weight) in the main product, respectively, and 0.756 (in grain size) and 0.065 (in protein ratio) in the secondary product, respectively. The highest and lowest values of heritability in the broad sense were 0.990 (for grain size) and 0.239 (for plot yield) in the main product, respectively, and 0.951 (for days to flowering) and 0.330 (for receptacle diameter) in the secondary product, respectively (Table 15).

Table 15: Broad $(\mathrm{H})$ and narrow $\left(\mathrm{h}^{2}\right)$ sense heritability values.

\begin{tabular}{lcccc}
\hline \multirow{2}{*}{ Characters } & \multicolumn{2}{c}{ First crop } & \multicolumn{2}{c}{ Second crop } \\
\cline { 2 - 5 } & $\mathrm{H}$ & $\mathrm{h}^{2}$ & $\mathrm{H}$ & $\mathrm{h}^{2}$ \\
\hline Seed yield (g) & 0.239 & 0.178 & 0.586 & 0.118 \\
1000-seed weight (g) & 0.466 & 0.059 & 0.863 & 0.390 \\
Days to flowering (day) & 0.936 & 0.331 & 0.951 & 0.387 \\
Days to physiological maturity (day) & 0.960 & 0.080 & 0.579 & 0.195 \\
Plant height (cm) & 0.747 & 0.287 & 0.607 & 0.433 \\
Head diameter (cm) & 0.556 & 0.202 & 0.330 & 0.284 \\
Oil content (\%) & 0.635 & 0.120 & 0.567 & 0.529 \\
Oleic acid (\%) & 0.849 & 0.346 & 0.864 & 0.194 \\
Linoleic acid (\%) & 0.823 & 0.315 & 0.786 & 0.265 \\
Palmitic acid (\%) & 0.354 & 0.094 & 0.440 & 0.281 \\
Stearic acid (\%) & 0.751 & 0.316 & 0.758 & 0.374 \\
Protein content (\%) & 0.629 & 0.119 & 0.654 & 0.065 \\
Hull percentage (\%) & 0.820 & 0.380 & 0.903 & 0.322 \\
Seed length (mm) & 0.990 & 0.810 & 0.954 & 0.756 \\
Seed width (mm) & 0.900 & 0.410 & 0.884 & 0.406 \\
Stem diameter (at the bottom) (cm) & 0.310 & 0.080 & 0.596 & 0.415 \\
Stem diameter (stem curve point) (cm) & 0.417 & 0.183 & 0.648 & 0.121 \\
\hline
\end{tabular}




\section{CONCLUSION}

In this study, cytoplasmic male sterile and restorer lines were used as parents and line $\times$ tester analysis was used as an appropriate method for the determination of general and specific combining abilities, various gene action types and heterosis (\%) and heterobeltiosis (\%) values of parents and hybrids in the development of suitable hybrid varieties in terms of high seed yield and oil content and other yield components in sunflower under both first and second crop production times. Significant differences were found among the hybrids and their parents. GCA effects were found to be highly significant for all traits. SCA effects, on the other hand, were non-significant for most of the traits. Based on GCA effects in the first and second crop production seasons, the inbreds $0043 \mathrm{cms}, 0046 \mathrm{cms}, 0195 \mathrm{cms}, 0583$ cms, $0704 \mathrm{cms}, 0708 R f, 0845 R f, 0951 R f$, and $1097 R f$ exhibited desirable GCA effects and were found to be good general combiners for most of the traits. These genotypes can be exploited by further breeding for developing superior genotypes and hybrids in sunflower.

\section{REFERENCES}

Arunachalam, V.C., 1974. The fallacy behind the use of the modified line $\times$ tester design. Indian J. of Genet. 34: 280-287.

Cochran, W.G., and Cox, M.C., 1957. Experimental design. John Willey and Sons, Inc. New York, NY.

Falconer, D.S., 1975. Introduction to quantitative genetics. Donald Press Co., New York, NY.

Fonseca, S. and Patterson, F.L., 1968. Hybrid vigor in a seven-parent diallel crosses in common winter wheat. Crop Sci. 8:85-88.

Griffing, B., 1956. Concept of general and specific combining ability in relation to diallel crossing systems. Aust. J. Biol. Sci. 9:227-232.

Hallauer, A.R. and Eberhard, S.A., 1966. Evaluation of synthetic varieties of maize for yield. Crop Sci. 6:423-427.

Hallauer, A.R. and Miranda, J.B., 1981. Quantitative genetics in maize breeding. Iowa State Univ. Press. Ames,

Hayes, H.K. and Immer, F.R., 1942. Methods of plant breeding. Mc Graw Hill Book Co., Inc. New York, NY.

Kempthorne, O., 1957. An introduction to genetic statistics. The Iowa State Uni. Press. Ames, Iowa, USA.

Rao, N.G.P., Rana, V.K.S. and Tripathi, D.P., 1968. Line $\times$ tester analysis of combining ability in sorghum. The Indian J. of Genet. 28(3): 231-238.

Singh, D.P. and Chaudhary, M.J., 1977. Line $\times$ tester analysis. In Biometrical methods in quantitative genetic analysis. p. 191-200.

Sprague, G.F. and Tatum, L.A., 1942. General vs. specific combining ability in single crosses of corn. J. Am. Soc. Agron. 34:923-932.

Steel, R.G.D. and Torrie, J.H., 1980. Principles and procedures of statistics. A biometrical approach. Mc Grow-Hill Book Co. New York.

Tan, A.S. 1993. Ayçiçeginde (Helianthus annuus L.) melez varyete $\left(\mathrm{F}_{1}\right)$ islahinda kendilenmiş hatlarin çoklu dizi (Line $\times$ Tester) analiz yöntemine göre kombinasyon yeteneklerinin saptanmasi üzerine araştirmalar. Doktora tezi. E.Ü. Zir. Fak. Fen Bil. Ens. Tarla Bit. Ana Bil. Dali. Bornova - Izmir.

Tan, A.S. 2000. Heterosis. Ege Tar. Ara. Enst. Yayin No. 96. Menemen, Izmir.

Tan, A.S. 2004. Kombinasyon Gücü. Bitki Islahi Egitimi (III). Tarim ve Köyişleri Bakanligi. Tarimsal Araştirmalar Genel müdürlügü. 13-17 Aralik 2004. Samsun.

Tan, A.S. 2005. Line $\times$ Tester Analizi. P 95-1 13. Bitki Islahinda Istatistik Genetik Metotlar. Ege Tar. Ara. Enst. No: 121. Menemen, Izmir.

Tan. A.S. 2009. Sunflower Research for Aegean Region of Turkey. Anual Report. Ministry of Agriculture, General Directorate of Agricultural Research. Menemen- Izmir, Turkey. 